\title{
Conocimiento y agricultura: \\ los agentes estatales de la modernización técnica en el Oasis Sur de Mendoza, Argentina, 1908-1920*
}

\author{
Knowledge and agriculture: \\ State agents of the technical upgrading in the Southern Oasis of \\ Mendoza, Argentina, 1908-1920
}

\author{
Florencia Rodriguez Vázquez*
}

RESUMO

En el presente trabajo procuramos realizar una reconstrucción de la trayectoria institucional de la Granja-Escuela del departamento de San Rafael (Mendoza), un proyecto del Gobierno provincial para generar y difundir conocimientos técnicos entre los agricultores que no tenían acceso a la Escuela Nacional de Vitivinicultura, esta última ubicada en la Ciudad de Mendoza. La iniciativa atravesó diversas dificultades para cumplir su objetivo fundacional, por lo que resulta interesante analizar, también, el desempeño del "agrónomo regional", una figura creada por el Ministerio de Agricultura de la Nación para capacitar sobre técnicas agrícolas a los agricultores que no tenían acceso a los sistemas de enseñanza formales. Buscamos, de este modo, establecer una relación entre la enseñanza de orientación técnico-productiva y una economía agroindustrial en un período de modernización.

PALAVRAS-CHAVE: Enseñanza agrícola; Modernización técnica; San Rafael (Mendoza).
ABSTRACT

In this paper we seek to make a reconstruction of the institutional trajectory of the GranjaEscuela department of San Rafael (Mendoza), a project of the provincial government to generate and disseminate knowledge among farmers who had no access to the Escuela Nacional de Vitivinicultura, the latter located in the city of Mendoza. The initiative went through various difficulties in meeting its objective foundation, so it is interesting to analyze also the performance of the regional agronomist, a figure created by the Agriculture Ministry's Office for training on agricultural techniques to farmers who had no access to formal education systems. Searching, thus establishing a relationship between the teaching of guidance technical-productive and agro-economy in a period of modernization.

KEYWORDS: Agricultural education, technical modernization, San Rafael (Mendoza).

\section{Introducción}

El proceso de modernización vitivinícola en Mendoza, desde fines del siglo XIX y principios del XX, ha sido estudiado en sus aspectos económicos (MARTÍN, 1992; RICHARD-JORBA y PÉREZ ROMAGNOLI, 1994; RICHARD-

\footnotetext{
* Este trabajo forma parte del proyecto "Entre dos crisis: transformaciones económicas, sociales, políticas y espaciales en la provincia de Mendoza (1890-1916)", dirigido por el Dr. Rodolfo Richard-Jorba y aprobado por el Consejo Nacional de Investigaciones Científicas y Tecnológicas (CONICET) (PIP 5.014). La autora agradece la especial contribución de la Lic. Andrea Cantarelli y del Centro de Medios Audiovisuales y Gráficos (MAGRAF) CCT Mendoza.
}

* Doutoranda da Universidad Nacional de Quilmes (UNQ) e Bolsista do CONICET / Argentina. 
JORBA, 1998; BARRIO, 2003; y RICHARD-JORBA, et. all, 2006), institucionales, políticos, legales (DÍAZ ARAUJO, 2006) y sociales (COZZANI, 1997; LACOSTE, 2003; y MATEU y STEIN, 2008). Sin embargo, la generación y difusión de conocimientos técnicos vitivinícolas que sustentaron este proceso han sido escasamente abordadas por la historiografía agraria argentina (GARGIULO y BORZI, 2004; FRESIA, 2005; y RODRÍGUEZ VÁZQUEZ, 2008a) y latinoamericana (BONFANTI, 2008).

Esto pese a que en los últimos años, hemos verificado una renovación temática que derivó en investigaciones sobre la enseñanza agrícola -para los niveles universitario, secundario y primario- en las regiones productivas pampeana (AUZA, 1996; GIRBAL-BLACHA, 1998; GUTIÉRREZ, 2000 y 2007; y GRACIANO, 2001 y 2004) y extrapampeanas (LENIS Y MOYANO, 2006; y RODRÍGUEZ VÁZQUEZ, 2008-b). Este trabajo se inscribe entonces en la perspectiva que vincula los planos educativo y productivo.

Los mismos coinciden en que la enseñanza agrícola funcionó, desde 1870, como fundamento del proyecto modernizador agroindustrial y socioeconómico del Estado Nacional, a través de la formación de recursos humanos profesionales, la instrucción de los agricultores, la contratación de técnicos extranjeros y el desarrollo de investigaciones útiles a cada economía regional objetivo para el cual se fundaron estaciones agronómicas y experimentales en la primer década del siglo XX-. Además, fue un recurso del Estado para “ (...) inducir a la población a adoptar determinados comportamientos económicos y decisiones productivas (...)" (GUTIÉRREZ, 2007: 27). La vida institucional de cada uno de estos establecimientos, su planificación académica, la designación de los profesores y del presupuesto anual así como el desarrollo de los estudios experimentales estuvo a cargo, primero, del Departamento Nacional de Agricultura y, luego, de su sucesor: el Ministerio de Agricultura de la Nación. En definitiva, estas escuelas estuvieron bajo jurisdicción del Estado Nacional por décadas, razón por la cual nos resultó interesante estudiar la Granja-Escuela de San Rafael (Mendoza), en tanto que fue un establecimiento íntegramente gestionado por el Gobierno provincial, por entonces, en manos de una elite "modernizante", descendiente de los pioneros de la vitivinicultura en Mendoza y “...sustentada en un grupo social integrado por propietarios, terratenientes, comerciantes o prestamistas, tal como ocurriría en el resto del país y en diversas 
regiones de América Latina" (RICHARD-JORBA, 1994: 69).

De este modo, nos interesa realizar una reconstrucción de la trayectoria institucional del mencionado establecimiento, cuál fue su impacto entre los agricultores locales y conocer si hubo una interacción con los establecimientos de orientación productiva dependientes del mencionado Ministerio, en este caso, la Escuela Nacional de Vitivinicultura -fundada en 1896- y su Estación Agronómica anexa -fundada en 1904-, las cuales reducían su radio de influencia a la Ciudad de Mendoza. De este modo, será factible preguntarnos sobre el surgimiento endógeno de una red que vehiculizara saberes en torno a la agricultura mendocina en tanto que alternativa válida, en primer lugar, para la dimensión cognitiva de la modernización industrial y, consecuentemente, la promoción de cambios tecnológicos locales o regionales.

En definitiva, indagaremos su rol como agente de capacitación de la masa de productores que se incorporaban masivamente a la agroindustria local y que no tenían acceso a la educación formal o superior. Dicho abordaje resulta incompleto si no nos preguntamos por la contribución de los agentes no institucionales de capacitación, así como también, por el posterior desempeño de la figura del agrónomo regional, una figura creada por el Ministerio de Agricultura de la Nación -en su reforma de la enseñanza agrícola de 1908- para la instrucción práctica de los agricultores.

\section{La enseñanza agrícola, fundamento del progreso nacional}

La formación teórica y práctica de recursos humanos para incorporarse a las industrias modernas y a las extensas zonas agrícolas que se reconvertían con miras a una explotación capitalista fue una preocupación primordial del Gobierno Nacional, junto con el tendido de líneas ferroviarias que garantizaran el comercio entre las zonas productoras y el mercado de consumo. Así, desde fines del siglo XIX la ligazón de las políticas educativas agrícolas y situación socioeconómica agraria fue una constante (GUTIÉRREZ, 2000: 6) dado que “...la producción agrícola constituyó una de las bases del extraordinario crecimiento económico ocurrido entre las postrimerías de los años 1870 y mediados de la década de 1910" (GIRBAL, 1992: 371).

Por esto, en el mencionado período, se crearon escuelas agrícolas espe- 
cializadas de acuerdo a las necesidades productivas de cada región; las mismas funcionaban bajo la órbita de organismos públicos nacionales, como el Ministerio de Agricultura o de Instrucción Pública. Por ejemplo, el presidente Domingo F. Sarmiento fundó la Escuela Nacional de Agricultura, en 1873, en la Ciudad de Mendoza. En general, fueron establecimientos con serias dificultades económicas, organizacionales y materiales para cumplir su misión (RODRÍGUEZ VÁZQUEZ, 2008 b). Algunos, incluso, fueron clausurados; otros, quedaron librados al abandono. Quizás por esto a mediados de la década de 1890 fueron refuncionalizados con miras a su reapertura y a una reorganización más eficiente, de acuerdo con los proyectos de promoción del perfil agrario argentino.

En 1896, la mencionada Escuela de Agricultura fue reinaugurada como Escuela Nacional de Vitivinicultura, convirtiéndose en pionera en la formación de recursos humanos desde principios de siglo XX en la región Cuyo. ${ }^{1}$ Los primeros años de la misma fueron afectados por constantes cambios de directores y de planes de estudio, cierres temporarios y por el precario estado de las instalaciones. Años después, en 1904, se creó la Estación Enológica -anexa al establecimiento-, la cual junto a una Bodega Modelo se dedicaban a la investigación y experimentación de las técnicas vitivinícolas y enológicas, con la materia prima obtenida en el plantel de vides existente en la Escuela -conformado por variedades europeas, traídas primero por el director Domingo Simois, y luego, por el enotécnico Leopoldo Suárez-, así como también, al análisis de muestras de vinos elaborados por los bodegueros locales. La efectiva concreción de los objetivos de ambas instituciones, al menos durante los primeros años de funcionamiento, fue un problema debido a las recurrentes crisis financieras y organizacionales que afectaban, cíclicamente, las tareas de generación y difusión de conocimientos (RODRÍGUEZ VÁZQUEZ, 2005 y 2006). Además, la resolución de las mismas demandaba mucho tiempo pues estaba supeditada a la División de Enseñanza Agrícola, una oficina de jurisdicción nacional que funcionaba en Buenos Aires. A su vez, las actividades de capacitación práctica o enseñanza

1 En la limítrofe provincia de San Juan -también, dedicada a la vitivinicultura- funcionaba una Escuela de Fruticultura o Quinta Agronómica, fundada por Domingo F. Sarmiento en agosto de 1862. El objetivo de su creación era el de convertirse en un centro de enseñanza técnica agronómica, introducir y experimentar con nuevas variedades de cultivos diversos (incluyendo vides) y atender a los problemas referidos a sanidad vegetal. Pero, a poco de su inauguración, fue casi abandonada y reinaugurada en 1880, en otro predio -donde funciona actualmente- y, a partir de 1939, cambió su nombre por el de Escuela de Fruticultura y Enología. 
extensiva de los trabajadores vitivinícolas era lo que primero se interrumpía.

Por esto, la opinión pública demandaba “(...) la implantación de un sistema racional de escuelas prácticas de agricultura que difunda la enseñanza agrícola elemental (...)”. ${ }^{2}$

Este contexto institucional complejo derivó en dos respuestas. Por un lado, la reforma general de la enseñanza agrícola -organizada también desde el Gobierno nacional-, en 1908, que proponía un nuevo organigrama para el desarrollo armónico de los establecimientos educativos agrícolas (universidades, escuelas especiales, escuelas prácticas) y contemplaba la enseñanza extensiva, de carácter práctico. ${ }^{3}$ Esto, a fin de consolidar un sistema integrado de enseñanza en sus diversos niveles, complementado con cursos prácticos temporales. El fundamento de esta reforma fue, asimismo, la revalorización de la región, "con el objetivo de optimizar la producción, en relación a mejoras científicas, mecanización, nuevas variedades de cultivos...” (GUTIÉRREZ, 2000: 4). La importancia de esta norma radicó, además, en su vigencia durante varias décadas (GUTIÉRREZ, 2007: 49).

Por otro lado, el Gobierno provincial -en manos de una élite modernizante, seguidora de las ideas alberdianas y sarmientinas-4 se sumó a la tarea de capacitación de los agricultores, a través de la inauguración de una Granja-Escuela en el departamento 5 de San Rafael.

\section{Estado provincial y educación productiva: el caso de La Granja- Escuela de San Rafael}

La provincia de Mendoza, en el centro oeste-argentino, se enmarca en la diagonal árida sudamericana, distinguiéndose dos oasis, el Norte -irrigado por los ríos Mendoza y Tunuyán- y el Sur -irrigado por los ríos Diamante y Atuel-, cuyo núcleo de mayor actividad económica y social se concentró en el

\footnotetext{
$2 \quad$ La Industria, $\mathrm{n}^{0}$ 751, Mendoza, 6/9/1910: 5 .

3 Los Andes, $\mathrm{n}^{0}$ 6.401, Mendoza, 4/11/1906: 4.

4 Juan Bautista Alberdi - político y publicista cuyo libro Bases y puntos de partida para la organización nacional fue una de las fuentes más importantes de la Constitución Nacional de 1853- fue defensor de un Poder Ejecutivo fuerte y promotor del progreso, entre sus objetivos ocupaba un lugar central el de promover la sociedad industrial a través de la inmigración. En tanto que para Domingo F. Sarmiento -Presidente de la Nación entre 1868 y 1874- el factor más importante de progreso era la escuela y la instrucción obligatoria (SANJURJO DE DRIOLLET, 2004).
}

5 “Departamento", en Mendoza, es la denominación que recibe la jurisdicción municipal. 
departamento de San Rafael.

\section{Mapa: Oasis Norte y Sur de Mendoza}

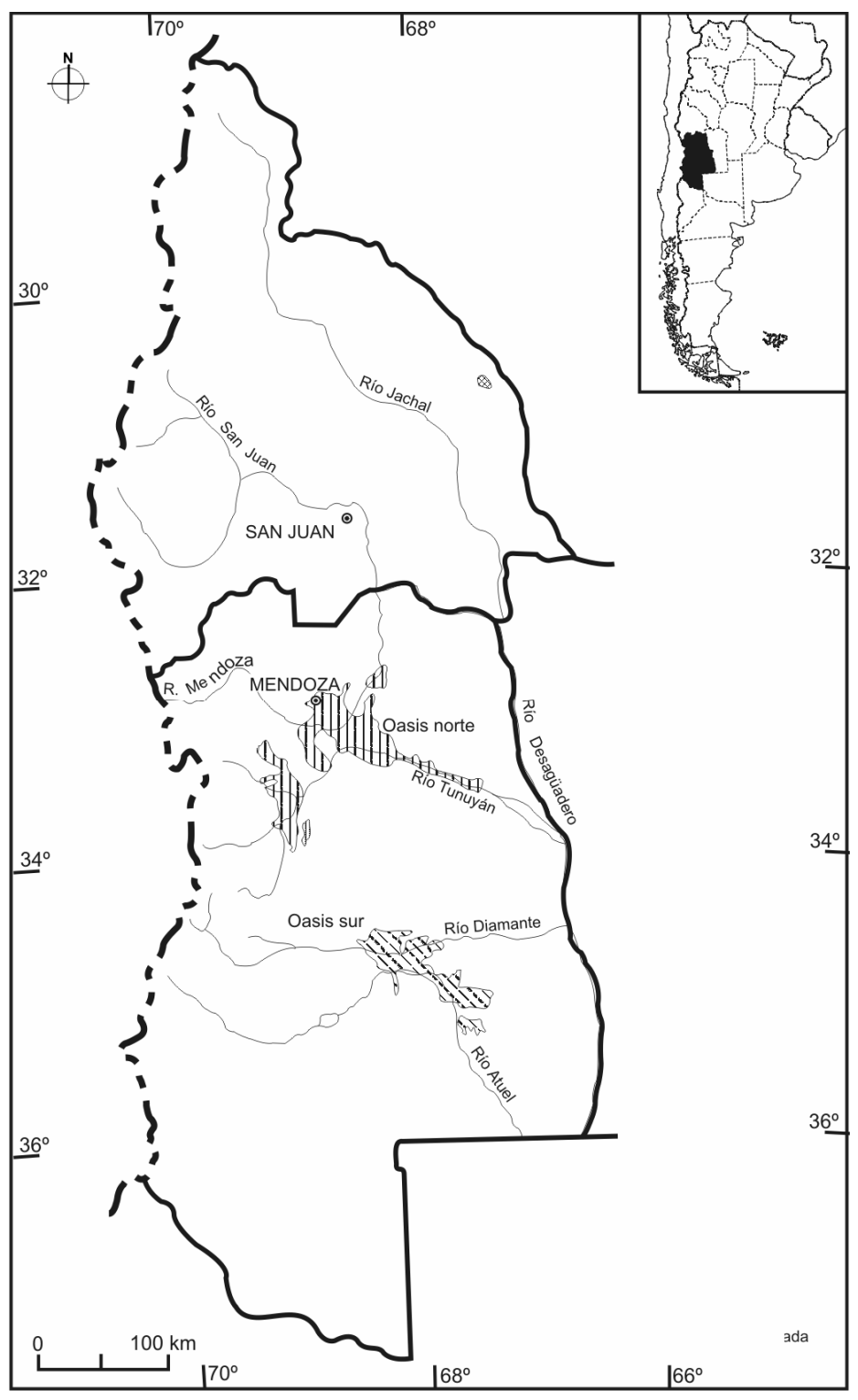

Nota: la delimitación de los oasis es aproximada.

Ambos espacios fueron transformados y refuncionalizados a partir de las decisiones económicas y políticas de la élite dirigente local que, en el último cuarto del siglo XIX, presentó un rol protagónico en la configuración de un modelo vitivinícola moderno, en sustitución de la exportación ganadera, impulsándolo como un sector casi excluyente de la economía provincial (RICHARD-JORBA, 1994: 95), aunque con una mayor incidencia en el Oasis Norte. Entre sus preocupaciones, se encontraban las medidas vinculadas al incremento agrícola productivo -como por ejemplo, los decretos de exención 
impositiva a los terrenos cultivados con vides- ${ }^{6} \mathrm{y}$ el tendido de una red ferroviaria que permitiera la conexión comercial con el mercado de consumo ubicado en el Litoral argentino; también proyectó un modelo productivo basado en la calidad, es decir, la elaboración de vinos finos que pudieran competir con sus pares europeos (RICHARD-JORBA, 1998). Las administraciones de los empresarios-políticos Francisco Civit (1873-1876), Elías Villanueva (1878-1881) y Tiburcio Benegas (1887-1889) fueron decisivas para el estímulo a la vitivinicultura, la transformación del oasis y la refuncionalización del espacio. En efecto, a partir de esos años se implantaron viñedos con criterios técnicos modernos y alta densidad de cepas por ha (3.000/4.00o), al finalizar el siglo XIX, 17.830 ha habían sido incorporadas a la viticultura a través de la promoción fiscal (CAMPI y RICHARD-JORBA, 2004: 55).

$\mathrm{El}$ ascendente crecimiento de las explotaciones cultivadas con vides y de la elaboración de vino, así como, el incremento del flujo comercial de vinos al Litoral se vio afectado por crisis económicas provinciales, la primera de ella en 1903 (BARRIO DE VILLANUEVA, 2003). Por ello, desde este grupo político se advirtió sobre los peligros del monocultivo de la vid y sobre la necesidad de impulsar nuevas industrias de base agrícola en la provincia. Razones que motivaron la inauguración de una Granja-Escuela en San Rafael (a 236 km, al Sur de la Capital mendocina), en 1908, a fin de difundir nuevas técnicas agrícolas. El gobierno local intervenía, de esta manera, en una actividad tradicionalmente dirigida por la Nación pero en la cual detectamos un vacío dado que había creado sólo una escuela práctica en la región de Cuyo, ubicada en San Juan.

De esto se desprende una nueva conclusión: la ubicación del establecimiento no fue azarosa sino una decisión estratégica.

Poco antes de su inauguración, Paul Pacottet -enotécnico francés contratado por el Ministerio de Agricultura de la Nación y por el Gobierno Provincialhizo un estudio sobre las condiciones de la tierra y de los diversos cultivos.7 Pacottet había sido traído por Pedro Benegas, hijo de Tiburcio, en 1908, para

6 El Poder Ejecutivo envió un proyecto a la Legislatura, finalmente aprobado, que clausuró el período de impulso a la radicación de viñedos a través de la exención impositiva, iniciada en 1881. En adelante, las nuevas plantaciones tendrían que pagar el impuesto establecido en la ley de Contribución Directa. La ley $\mathrm{N}^{\circ} 245$ del 27-9-1902 (Registro Oficial Provincia Mendoza R.O.P.M.-, 1902, t. III: 74-76) derogó la última ley (del 3-10-1895) de exención impositiva (BARRIO DE VILLANUEVA, 2006).

7 Album Argentino Gloriandus, número extraordinario dedicado al señor Gobernador Dr. Emilio Civit, Mendoza, 1910, f. 12 v. 
enseñar técnicas enológicas de avanzada. De tal visita, tres años después, el francés publicó Vinificación en la Provincia de Mendoza (República Argentina) (RICHARD-JORBA, 2004). Resulta oportuno mencionar que este fue un caso de migración temporaria con la finalidad específica de transferir conocimientos y procedimientos técnico-enológicos, y que luego se habría convertido en definitiva dado que entre 1911 y 1916 realizó sucesivas compras y ventas de terrenos cultivables y chacras del "pueblo Monte Comán" (San Rafael). Por ejemplo, en 1916 vendió un terreno de 400 ha, que había adquirido en $1911 .^{8}$ Esto manifiesta, además, que el técnico no se habría dedicado exclusivamente a las investigaciones agrícolas como empleado del ministerio sino que se habría volcado a los negocios de bienes raíces. Asimismo, marca una similitud con otros expertos extranjeros que hicieron el mismo recorrido profesional, como es el caso del agrónomo ruso Aaron Pavlovsky, director de la Escuela Nacional de Agricultura de Mendoza entre 1884 y 1888. Esto confirma que para la implantación de un nuevo modelo, no sólo se necesitaba capital sino también conocimientos científicos y técnicos que pudieran ser adaptados a las especiales condiciones climatológicas, ambientales y edafológicas de la provincia. Por ello, se contrataron a especialistas europeos para que realizaran investigaciones y difundieran sus conocimientos que, luego, fue necesario interpretar a la luz de las condiciones locales.

El informe realizado por el enotécnico manifestó las calidades óptimas de los terrenos para encarar diversos cultivos, situación que confirmaban las estadísticas: en 1910, había 2.403 ha plantadas con viñas francesas, criollas y, lo más destacable, 38 ha para el cultivo de variedades especiales9. Además, San Rafael ya se perfilaba como una zona próspera que podría hacerle sombra a los departamentos más pujantes del Oasis Norte de la provincia, a raíz de la inauguración del ramal ferroviario (1903), la llegada masiva de inmigrantes y la actividad económica: en 1907 se contabilizaron más de 12.000 habitantes, ${ }^{10}$ con un gran porcentaje de inmigrantes franceses e italianos y, entre 1908 y 1911, se registró el inicio de la actividad de un gran número de bodegas, a partir de la ayuda financiera de los bancos (CANTARELLI, 2006). De este modo, el contexto de

\footnotetext{
8 Archivo General Provincia Mendoza (en adelante, A.G.P.M.), Protocolo Notarial, escritura $\mathrm{n}^{\mathrm{o}}$ 52, 26/12/1916. Contrato celebrado entre Pablo Pacottet y Pablo Patter, f. 1.391.

9 Album Argentino..., op. cit., f. 59 v.

10 Ibidem, f. 5 v.
} 
prosperidad socioeconómica, demográfica y productiva -que se integró más tardíamente a la vida política y económica provincial, y que la convirtió luego en "la región del porvenir"-11 justificaba la instalación de la Granja-Escuela.

Entendemos a esta última, por un lado, como un nuevo intento de la élite provincial por dirigir la generación y difusión de conocimientos útiles a la agroindustria $^{12} \mathrm{y}$, por el otro, como una respuesta a las demandas de reestructuración de la enseñanza agrícola para el fortalecimiento de las escuelas prácticas regionales o locales. Al año siguiente, la Dirección General de Industrias ${ }^{13}$ provincial sentó las bases para la fundación del establecimiento en el distrito de Monte Comán (Colonia Francesa) ${ }^{14}$, en un terreno de 4 hat ${ }^{15}$ donado, en 1900, por el inmigrante francés Rodolfo Iselín -activo colonizador, empresario ganadero y vitivinícola - para la instalación de un vivero y quinta de ensayos. ${ }^{16}$ El gobierno provincial se comprometía a destinar $\$ 3.000$ para la instalación de la misma y $\$ 50$ mensuales para su mantenimiento.

Así, entre la donación e inauguración de la Granja-Escuela, hubo diversas propuestas acerca del destino que se le daría a los terrenos. Primero, se pensó en construir un vivero ya que contaba con " 50 variedades en plantas forrajeras perennes y otras tantas maderables" ${ }^{17}$ Por último, el diario local proponía la conveniencia de instalar allí mismo una estación meteorológica, con miras a prevenir los accidentes climáticos. ${ }^{18}$ No obstante, ninguno de estos proyectos prosperó -en 1903 aún no se designaba un director técnico para la misma-,19 razón por la cual se iniciaron las prédicas para instalar allí una escuela agrícola

11 El diario local Los Andes señalaba en 1908 "región que hoy llama la atención de toda la república, y donde fluyen brazos trabajadores y capitales que se invierten en canales de riego de grandes extensiones y en cultivos intensivos" (Apud SANJURJO DE DRIOLLET, 2007: 235).

12 R.O.P.M., Decreto 11/1/1887, p. 237. Entre 1887 y 1890 la élite provincial se hizo cargo de Escuela Nacional de Agricultura, dado que el Gobierno Nacional se había desprendido de ella por carencia de recursos financieros.

13 La Tarde, $\mathrm{n}^{0}$ 11, Mendoza, 14/6/1910: 7.

14 El grupo de inmigrantes franceses allí instalados gozaron de una situación financiera favorable que les permitió aplicar sus conocimientos sobre vitivinicultura y traer maquinarias para construir bodegas. También fomentaron el cultivo de frutales (SANJURJO DE DRIOLLET, 2006: 257).

15 Ecos de San Rafael, Mendoza, 16/9/1900: 1.

16 R.O.P.M., 20/9/1900: 260.

17 Ecos de San Rafael, 22/1/1902, ${ }^{\circ}$ 166: 1.

18 Ibidem, 28/10/1900: 1.

19 Ibidem, 16/9/1903, $\mathrm{n}^{\circ}$ 252: 1. 
o un vivero departamental, para la propagación de árboles frutales y hortalizas ${ }^{20}$ a través de la venta de semillas y ejemplares a un bajo costo. Incluso, en 1904 la Municipalidad propuso hacerse cargo de los ensayos ${ }^{21}$, "que hasta el momento, no han suministrado nada práctico". ${ }^{22}$ Prédica que sólo a partir de 1908 tuvo asidero, con el proyecto de Emilio Civit -gobernador de Mendoza, por segunda vez, desde 1907 a 1910- de fundación de una Granja-Escuela en San Rafael y una chacra de experimentación de cultivos y criadero de plantas anexos.

Su principal objetivo era "dar enseñanza práctica manual directa de la agricultura intensiva en general y, en especial, del cultivo de árboles frutales y de la elaboración de los productos”. ${ }^{23}$ Esto para formar un personal idóneo de capataces agrícolas -“de los que carece la provincia” -“24 en un curso breve de 2 años de duración; en el cual también recibirían instrucción primaria -debido a la, por entonces, escasa difusión de la educación. El título de capataz industrial exigía un año más de cursado, "destinado a la enseñanza de la recolección, preparación, elaboración y tratamiento industrial de los frutos y sus derivados". 25 Para ello, el establecimiento disponía de un terreno de 40 ha, ${ }^{26}$ destinado al trabajo en diversas secciones (agrícola, ganadera, industrial), talleres y un museo, permitiendo la explicación y la demostración directas. Así, no poseía un plan de estudio específico sino cursos de explotación agrícola.

Advertimos, así, que el establecimiento no se dedicaría exclusivamente a la promoción de la vitivinicultura en un terreno que ya se consideraba promisorio para la actividad, sino que fomentaba diferentes agroindustrias. Esto era consecuente con el pensamiento de la élite acerca de la inconveniencia del monocultivo de la vid para la economía regional y con la intención del Ministerio de Agricultura de proveer una enseñanza agrícola basada la introducción de mejoras científicas.

Esta orientación técnico-práctica funcionaría, a su vez, como complemento ideal de la Escuela Nacional de Vitivinicultura, no sólo porque se dedicaría a

20 Ibidem, varios números de 1901.

21 Ibidem, 27/1/1904, $\mathrm{n}^{\circ}$ 270: 1.

22 Ibidem, 3/2/1904, $\mathrm{n}^{\circ}$ 271: 1.

23 Registro Oficial de la Nación. Decreto del 13/12/1908. El subrayado es nuestro.

24 R.O.P.M. Decreto formulando plan de enseñanza. 13/12/1909: 385.

25 El Debate, no 6.331, Mendoza, 1/2/1910: 3.

26 Nótese que superaba ampliamente la superficie del campo de cultivos de la Escuela Nacional de Vitivinicultura, de 27 ha. 
otra rama productiva sino también porque esta última era considerada una Escuela especial, por lo que tenía "un ingreso más restrictivo y formaba técnicos para las grandes empresas agropecuarias o funcionarios del Ministerio de Agricultura” (GUTIÉRREZ, 2000: 4).

Asimismo se esperaba que cumpliera un efecto poblatorio de la Colonia Francesa, ya que la capacitación de los jóvenes en las tareas agrícolas traería como consecuencia “...el hábito y la capacidad para el trabajo rural, que sólo se adquiere por la enseñanza...”(M.A.N., 1908: 30) y el asentamiento definitivo de la familia en las zonas rurales. Incluso antes de la inauguración oficial del establecimiento, así se refería un diario local: “...será el exponente del movimiento rápido de población de Monte Comán, tanto por los beneficios que se cifran hará el mismo a esta región, como por los rumbos del todo adoptable, que le imprimirá el Sr. Ciranna...”. ${ }^{27}$ Esto nos presenta otra estrecha relación con la mencionada reforma de la enseñanza agrícola que manifestaba que las escuelas prácticas vendrían a restituir “...el equilibrio entre la población rural y urbana, roto en nuestro país por la poderosa atracción y (...) fascinación, que ejerce nuestra gran metrópoli” (M.A.N., 1908: 30).

Los primeros años: proyectos y conflictos

Los medios gráficos lo anunciaron como el primer establecimiento de este tipo en el país. De acuerdo con la mencionada reforma de la enseñanza, esta sería una escuela práctica, pero gestionada por el Gobierno provincial.

El primer director designado fue el agrónomo Camilo Ciranna -jefe de cultivos de la chacra experimental de San Juan-,28 quien se radicó definitivamente en San Rafael. En efecto, en 1914 aparece como propietario de una bodega. ${ }^{29}$ Observamos, así, la vinculación entre los capitales cultural y productivo.

En sus primeros años, la escuela tuvo a su cargo 12 alumnos que recibirían un jornal -proporcional al trabajo que efectuaran- de hasta \$2 diarios, por lo que se asociaba a la figura del "peón-alumno" difundida en algunas escuelas prácticas de la provincia de Buenos Aires (GUTIÉRREZ, 2007: 64). Un

\footnotetext{
27 La Tarde, 14/6/1910, $\mathrm{n}^{\circ}$ 11: 7.

28 Boletín Oficial República Argentina (en adelante B.O.R.A.), XV, $n^{\circ}$ 4.218, Dto 5/12/1907: 1.104 .

29 Elaboraba un vino marca "Ciranna". B.O.R.A., 13/4/1914.
} 
porcentaje se destinaba al ahorro en el Banco de la Provincia y sería entregado al alumno para que lo invirtiera en un emprendimiento agrícola propio, esto sólo si finalizaba el curso, de lo contrario, el beneficio caducaba. ${ }^{\circ}$ Esto se financiaría, en parte, con la venta de árboles, plantas y semillas en las instalaciones propias.

Se dirigía, de este modo, el acceso a la instrucción de los jóvenes con menos recursos. Los hijos de familias de la élite local, interesados por los estudios agrícolas, eran enviados a la Escuela Nacional de Vitivinicultura, tal es el ejemplo de José Salas, descendiente de una familia relacionada con la vitivinicultura. Esto concuerda con lo advertido por Gutiérrez acerca de que la enseñanza agrícola estaba dirigida a distintos sectores sociales, con finalidades específicas (GUTIÉRREZ, 2007: 54). La imposición de un entrenamiento práctico en las labores agrícolas evitaría, además, que los graduados intentaran ingresar a la universidad o emigrar a la ciudad.

También se proponía difundir entre los agricultores los resultados de las investigaciones propias, a fin de mejorar los procedimientos técnicos y económicos ${ }^{31}$ que demandaban las tareas agrícolas.

Sin embargo, en 1910 se denunció que aún no abría ${ }^{2}$ y que el escaso personal contratado permanecía impago.33 Quizás por esto, los senadores nacionales de Mendoza, Emilio Civit y Elías Villanueva, 34 y sus pares de Buenos Aires, Manuel Láinez y Benito Villanueva presentaron un proyecto para instalar un establecimiento similar en Cuadro Nacional -otro distrito de San Rafael-: "Confío en que la transformación de un predio, hasta hoy abandonado, en un predio excepcionalmente agrícola (...)”.35

No sabemos si esta iniciativa prosperó, sólo hemos confirmado que en 1911 la Escuela funcionaba, bajo la dirección de Carlos Tabanera, ${ }^{36}$ pues se entregaron 20 becas de estudio. ${ }^{37}$ Aunque sólo ocupó su cargo por un año,

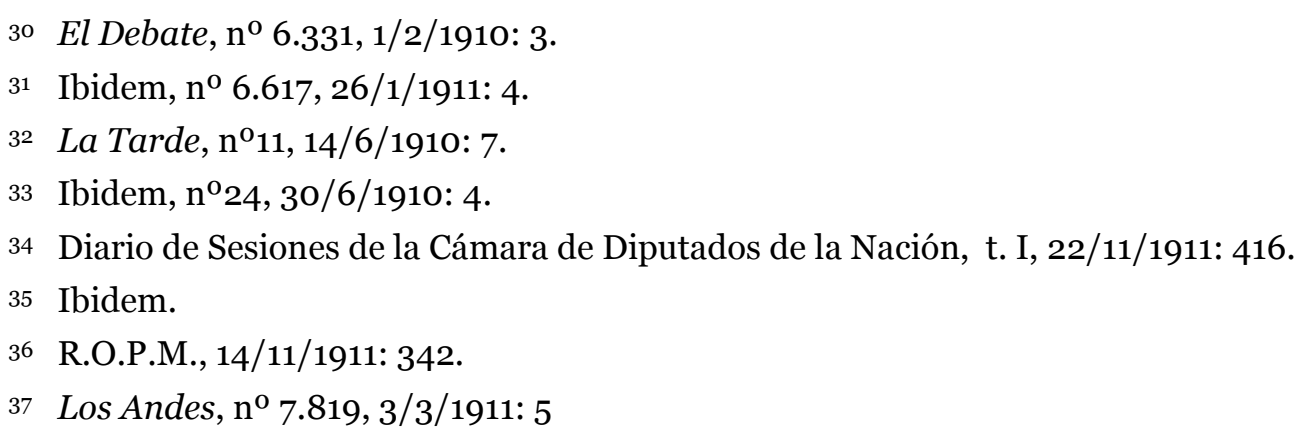


reemplazándolo el enólogo José Gomensoro, ${ }^{38}$ figura pública a destacar dado que habría sido alumno de la Escuela Nacional de Vitivinicultura, becado por el gobierno provincial para realizar sus estudios y luego para perfeccionarse en Conegliano.39 Poco después, también registramos su labor en la Dirección General de Industrias provincial. ${ }^{40}$ Resaltamos su gestión por dos motivos, uno, confirma la concreción del proyecto del gobierno provincial acerca de la importancia de instaurar un sistema de becas de perfeccionamiento en el extranjero para los graduados de la Escuela, quienes a su regreso deberían prestar su servicio en la provincia y en Argentina. Por otro lado, nos permite valorar el aporte de la Escuela Nacional de Vitivinicultura a la agricultura mendocina, a través del desempeño de sus graduados en el ámbito público.

Además, esta fue el único docente de la Granja con formación probada en ciencias agronómicas. Las fuentes revelan que no se tuvo en cuenta los antecedentes profesionales o académicos para la designación del resto de los profesores, ${ }^{41}$ tal como aparece en el siguiente cuadro:

Directores de la Granja-Escuela de San Rafael (1908-1920)

\begin{tabular}{|c|c|c|}
\hline Director & $\begin{array}{c}\text { Formación } \\
\text { profesional }\end{array}$ & $\begin{array}{c}\text { Antecedentes en } \\
\text { agricultura/ } \\
\text { vitivinicultura }\end{array}$ \\
\hline Camilo Ciranna & $\mathrm{s} / \mathrm{d}$ & Vitivinicultor \\
\hline Carlos Tabanera & $\mathrm{s} / \mathrm{d}$ & $\mathrm{s} / \mathrm{d}$ \\
\hline José Gomensoro & $\begin{array}{c}\text { Enólogo. Escuela } \\
\text { Nacional de } \\
\text { Vitivinicultura } \\
\text { (Mendoza) y Escuela de } \\
\text { Conegliano (Italia) }\end{array}$ & $\mathrm{s} / \mathrm{d}$ \\
\hline José Castro & $\mathrm{s} / \mathrm{d}$ & $\begin{array}{c}\text { Ex Jefe de Cultivos } \\
\text { (Granja-Escuela) }\end{array}$ \\
\hline
\end{tabular}

Fuente: Elaboración propia sobre la base de Registros Oficiales de la Provincia de Mendoza (1905- 1920), revista La Vitivinicultura Argentina (1910-1911) y diario La Tarde (1916).

Marcadas estas cuestiones, debemos ahora mencionar que el normal

38 R.O.P.M., 15/3/1912: 395.

39 R.O.P.M.1906, Dto. 14/07/1906: 197; R.O.P.M. 1908, 23/07/1908: 206.

40 R.O.P.M., 13 y 14/7/1914: 446. B.O.P.M., Dto 11/2/1915: 6.368.

${ }^{41}$ A modo de ejemplo, señalamos el nombramiento de Ernesto Muñoz como Jefe de Cultivos (R.O.P.M., 23/1/1912: 125), de quien no tenemos datos sobre su formación profesional. 
ejercicio del establecimiento departamental fue afectado por sucesivas interrupciones, pese a las prometedoras intenciones anunciadas. Así, atravesó dificultades similares a las de la Escuela Nacional de Vitivinicultura: sueldos impagos y demoras para completar el plantel de los profesores.

Esto perjudicó a la institución como agente de transferencia, pero las mayores falencias se verificaron en la función de generación de saberes, pese a que había sido creada para extender los espacios de ensayos de los cultivos. Así lo criticaba la prensa mendocina:

(...) estamos en el primer peldaño respecto de algunos cultivos muy importantes [debido] a que no se han propagado establecimientos de enseñanza que se denominan granjas modelo y experimentales que tan positivos resultados dan para la industria, (...) tendientes a extirpar la rutina secular y el empirismo que todavía reina aquí(...). 42

Así, en 1913 otro diario local denunciaba que “(...) [la Granja-Escuela] de nada ha servido ni servirá en manos de una administración como la actual que rige los destinos de la provincia”. 43 Ese mismo año fue cerrada -pese a que el gobierno había asignado una subvención de \$300-44 y aún en 1916 no era reabierta (AMADEO, 1916: 74). Sólo en 1919 se intentó recuperarla sobre la base de una cooperativa administrada por el gobierno y sostenida por los productores. 45 No obstante, no pudimos confirmar su ejecución.

Esto marca una diferencia fundamental con la Escuela Nacional de Vitivinicultura y la Estación Enológica, que lograron sortear estas dificultades con miras a generar un capital técnico local. Probablemente gracias al financiamiento nacional y a que era la única escuela especializada en formación vitivinícola.

En conclusión, la creación de la Granja-Escuela para la capacitación práctica de los vitivinicultores fue la manifestación de los ideales de la élite local con miras, por un lado, al fomento de diversas agroindustrias (olivicultura, fruticultura) en el Oasis Sur de la provincia de Mendoza y, por el otro, la población y asentamiento definitivo de la familia en ese espacio rural. No obstante, una vez más, la puesta en marcha de ese proyecto fue desviada por las realidades materiales adversas y la desorganización administrativa que afectó al establecimiento desde su fundación, en una etapa en la que “...no era fácil

\footnotetext{
42 Los Andes, $\mathrm{n}^{0}$ 7.868, 30/4/1911: 8.

43 La Industria, $\mathrm{n}^{\circ}$ 1.553, 28/5/1913: 4.

44 R.O.P.M., 31/10/1913: 282- 283.

45 Los Andes, $\mathrm{n}^{0}$ 11.045, 9/11/1920: 5.
} 
constatar resultados positivos e inmediatos en la instrucción agrícola que pudieran volcarse en el progreso rural de la región de asentamiento de las escuelas..." (GUTIÉRREZ, 2007: 82).

\section{El agrónomo regional}

Si bien los aportes de la Granja-Escuela a la generación y difusión de conocimientos entre los vitivinicultores fueron escasos, las posibilidades de capacitación y modernización agroindustrial no se agotaron allí. En efecto, en 1908 el Ministerio de Agricultura de la Nación, convencido de que el Estado debía "velar por la instrucción agrícola del individuo rural que no puede concurrir a la escuela, sea porque su edad, ocupación diaria o preparación no lo permita..." (M.A.N., 1908: 39), creó la figura del "agrónomo regional”, en consonancia con el espíritu de la reformada ley de enseñanza agrícola que impulsaba la enseñanza extensiva.

La misma destacaba entre sus actividades (M.A.N., 1908: 46): cursos para niños y adultos; cátedras ambulantes; servicio de informaciones; experiencias cooperativas; concursos y exposiciones regionales; estimulos a las asociaciones y sindicatos agrícolas; y organización de los agrónomos regionales

El "agrónomo regional" cumplía la función de difundir los conocimientos agrícolas entre los trabajadores a fin de mejorar sus cultivos, seleccionar semillas y combatir las plagas. Esto, a través de la oferta gratuita de cátedras ambulantes -es decir, los profesionales se trasladaban hasta las plantaciones o establecimientos productivos para instruir a los agricultores-, conferencias los días feriados, servicio de consultas en su oficina, promoción de experiencias cooperativas, organización de concursos y exposiciones. 46 Estas actividades serían planeadas de acuerdo a las necesidades de las diferentes zonas agrícolas del país, así constituían un aporte insoslayable para los vitivinicultores y agricultores mendocinos, pues la prensa lamentaba:

\footnotetext{
Ellos cultivan la tierra empíricamente, con los procedimientos primitivos (...) sobretodo, lo que hace falta es inculcar los principios de previsión para que puedan luchar ventajosamente contra los contratiempos frecuentes en las faenas campestres. 47
}

\footnotetext{
46 Almanaques del Ministerio de Agricultura, 1927: 16.

47 La Industria, $\mathrm{n}^{0}$ 1.251, 21/5/1912: 5.
} 
En Mendoza, el primer "agrónomo regional" designado se estableció en San Rafael y fue el enólogo Enrique Silvetti, graduado de la Escuela Nacional de Vitivinicultura. A continuación, demostraremos cómo esta figura marcó un especial punto de contacto con la mencionada Escuela y evaluaremos su contribución para la capacitación práctica de los agricultores locales. En primer lugar, habría cumplido una importante labor entre quienes no podían asistir a la Escuela de Vitivinicultura -alejada de las zonas agrícolas- o a la GranjaEscuela, por medio de cursos temporarios. De este modo, lo consideramos un agente de transferencia tecnológica que marcó un vínculo entre un centro de experimentación y generación de conocimientos vitivinícolas y enológicos locales y la masa de los productores, distribuida por toda la provincia. A partir de 1912 encontramos avisos -aunque esporádicos- en distintos medios gráficos, de circulación provincial, que promocionaban su actividad y los informes que comentaban los resultados de estas experiencias: consultas recibidas en su oficina, consejos a los viñateros afectados por el granizo -principalmente, recomendando la poda de las cepas mutiladas-, conferencias sobre los modernos sistemas de cultivos y cómo combatir las plagas, inspecciones en viñas, frutales y bodegas. ${ }^{48}$ Estas actividades permitían el acceso a información actualizada -generalmente publicada en revistas especializadas- a aquellos productores analfabetos, o bien, inmigrantes que no dominaban el español.

Su labor se restringía a los distritos cercanos a esa ciudad: Rama Caída, Cañada Seca, Monte Comán y Cuadro Nacional. Sólo esporádicamente visitaba los departamentos más alejados debido a la carencia de un coche-escuela, en general, donado por las empresas ferroviarias. Del mismo dispuso desde 1916, lo que habría facilitado la cobertura de más zonas agrícolas de la provincia. Otra dificultad residía en que la mayoría de los profesionales designados debían cubrir dos provincias (MAROTTA, 1916: 15), en este caso, aún no hemos comprobado si debía trasladarse hasta alguna zona limítrofe.

$\mathrm{Su}$ aporte fue muy fructífero porque ampliaba el radio de acción de la Escuela de Vitivinicultura y la Estación Agronómica, principalmente en actividades de divulgación técnica entre productores vitícolas, frutícolas y hortícolas y, en menor medida, en las bodegas -de las 89 bodegas registradas en 1913, 51 tenían una capacidad de elaboración de hasta 1.000 hl anuales, es decir,

${ }_{48}$ La Tarde, $\mathrm{n}^{\circ}$ 898, 30/5/1913: 4. 
que eran considerados pequeños y medianos establecimientos vitivinícolas dirigidos, generalmente, por sus dueños, con escasa preparación técnicoenológica.49 Además, corroboramos la presencia sucesiva de artículos periodísticos fundados en estudios locales, por ejemplo, la experimentación de nuevos sistemas de poda en las vides y frutales. Así, también funcionó como agente de investigación y generación de conocimientos útiles para la producción agrícola en el Oasis Sur, tarea que nos permite revalorizar el papel de los técnicos agrícolas formados en las escuelas y universidades argentinas en la modernización agroindustrial, como lo ha señalado Girbal para el caso de los ingenieros agrónomos en la región pampeana argentina (1992: 375).

Nos resulta extraño que no haya trabajado en cooperación con la GranjaEscuela de San Rafael, tal vez una oportunidad perdida para su reapertura o reorganización eficiente. Esto denota la desconexión entre instituciones con finalidades similares pero dependientes de distintas jurisdicciones.

\section{La difusión informal de conocimientos}

Además de los circuitos institucionales de formación de recursos humanos y capacitación práctica sobre conocimientos agrícolas, debemos tener en cuenta los espacios informales de divulgación de técnicas agrícolas.

En primer lugar, mencionamos a la prensa especializada. A partir de 1904, y como consecuencia de una crisis vitivinícola provincial ocasionada, entre otros motivos, por la escasez de conocimientos científicos generados localmente (ARATA, 1903), registramos un boom editorial en Mendoza. Algunos de los profesores y alumnos de la mencionada Escuela de Vitivinicultura se propusieron la publicación de revistas que abordaban temáticas agrícolas y que respondían a las consultas específicas de los agricultores. No obstante, presumimos la dificultad de estas publicaciones para conectarse asiduamente con los agricultores del Oasis Sur, situación que se habría modificado a partir de 1911 con la revista Vinicultura Práctica, editada por Juan Canadé, un enólogo radicado en San Rafael por sus negocios de representación de bodegas y destilerías, departamento al que representó en 1923 ante el Centro Vitivinícola

49 Anuario de Mendoza, 1913: 235. 
Nacional y en donde, además, instaló una bodega en 1926.50 Asimismo, contaba con experiencia en la tarea editorial ya que había sido colaborador en la Revista Vitivinícola Argentina. Fue un caso especial de agente de difusión estrechamente vinculado al sector privado. En efecto, no hemos hallado indicios de su formación académica ni de su vinculación con la Escuela Nacional de Vitivinicultura ni con el Ministerio de Agricultura de la Nación; no obstante, ejerció una importante capacitación a través de las mencionadas publicaciones.

Su labor de divulgación se concentró en temas enológicos, útiles para los bodegueros que buscaban mejorar la elaboración. Así lo expresaba en uno de sus números:

Vinicultura Práctica es el deseo de dar mayor impulso a su programa de difundir la mayor suma de conocimientos sobre la vinificación racional entre los industriales que no hayan tenido acceso a los libros, los laboratorios y las bodegas experimentales (...) (CANADÉ, 1912: 3).

Por otro lado, debemos tener en cuenta el "efecto demostración" ejercido entre los agricultores. Los asistentes a las conferencias y charlas dictadas por el agrónomo regional, aunque fueran un número escaso, luego divulgarían las técnicas aprendidas entre sus pares. En el caso de la vitivinicultura, RichardJorba (2003) ha señalado el rol protagónico de los contratistas de viña, cuyas formas de plantación y conducción de las vides, densidad de cepas plantadas por hectárea y técnicas de poda fueron imitadas por sus pares. A su vez, detectamos varios casos de cultivos experimentales realizados por empresarios locales, aunque vinculados a la vitivinicultura. Los mismos fueron un modelo para la posterior difusión entre sus vecinos.

A modo de ejemplo, en 1902, un especialista, el Ing. Luciano Garolla, recomendaba la importancia de experimentar diferentes variedades de vides y de poda y tomaba el ejemplo de los ensayos realizados por "los señores Tiburcio Benegas e hijo, en la margen derecha del río Diamante" (GAROLLA, 1902: 2).

Por su parte, en 1911, el enólogo Leopoldo Suárez señalaba en su libro sobre ampelografía mendocina las experiencias realizadas por Rodolfo Iselín con la variedad tannat en sus viñedos de San Rafael (SUÁREZ, 1911: 184), hasta entonces sólo cultivada, con fines experimentales, en la Escuela de Vitivinicultura.

La especial atención dada por estos actores a la vitivinicultura -estudios

50 Dirección General de Industrias, Fiscalización y Consumo, Mendoza, expediente $\mathrm{n}^{\circ}$ 1.747, 4/2/1926. 
especiales, experiencias y ensayos con diversas variedades, vinculación comercial con la venta de vinos- fue, en definitiva, el reflejo de la supremacía alcanzada por el modelo productivo y económico a principios del siglo XX en Mendoza.

\section{Conclusiones}

La capacitación práctica de los agricultores y de los vitivinicultores, fundamental para la innovación técnica y la modernización productiva de las extensas áreas del Oasis Sur, fue un proceso lento y complejo. La misma se convirtió en un objetivo expreso del Estado provincial, el cual buscó dotar de un establecimiento agrícola a un espacio potencialmente próspero pero que aún no era contemplado por el Gobierno nacional en sus planes de enseñanza.

Proyecto provincial que sufrió múltiples carencias financieras $\mathrm{y}$ profesionales, así como dificultades organizacionales, las cuales entorpecieron, hasta paralizar, los ensayos, experimentación y enseñanza de los jóvenes de escasos recursos que, se pretendía, se establecieran en ese espacio rural.

Una alternativa válida fue la llegada del "agrónomo regional" a San Rafael, ampliando las áreas de difusión de la única escuela agrícola especializada de la provincia y constituyendo un real puente entre el avance de los conocimientos técnicos generados localmente y los agricultores. La divulgación no se redujo a conocimientos vitivinícolas y enológicos sino que promovió el cultivo de hortalizas y frutales, y la arboricultura, de acuerdo con la intención de la élite provincial de incentivar diversos cultivos en la provincia.

No obstante lo cual, la ganadería y la vitivinicultura fueron las actividades económicas predominantes en el departamento hasta 1930 (GUIBOURDENCHE DE CABEZAS, 1956), sólo suplantadas a partir de la década de 1940, cuando se dio un proceso de diversificación industrial que consolidó, también, a otras actividades de base agrícola y a sus industrias derivadas -conservas, desecación, entre otras- (MARTíN, 1992:173-194).

\section{Bibliografía}

Almanaques del Ministerio de Agricultura, Sección propaganda e informes. Buenos Aires: Talleres Gráficos, 1927. 
AMADEO, Tomás. La enseñanza y la experimentación agrícolas en República Argentina. Buenos Aires: Talleres Gráficos del Ministerio de Agricultura, 1916.

Anuario de la Dirección General de Estadísticas de la provincia de Mendoza correspondiente a 1913. Establecimiento gráfico "La Tarde", 1914.

AUZA, Néstor. La enseñanza agraria del centenario a 1930. IN: Noveno Congreso Nacional y Regional de historia Argentina, Rosario, 1996 (separata).

BARRIO DE VILLANUEVA, Patricia. Hacia la consolidación del mercado nacional de vinos. Modernización y desarrollo del sector vitivinícola de Mendoza (1900-1914). Revista Espacios, UNPA, año IX, 2003, nº 26.

. Una crisis de la vitivinicultura en el Oeste argentino (Mendoza) a principios del siglo XX. América Latina en la Historia Económica, México, Instituto Mora, julio-diciembre de 2006, $\mathrm{n}^{0} 26$.

BONFANTI, Daniele. La segunda generación. Traspaso generacional, continuidades y cambios en una empresa vitivinícola uruguaya (1920-1945), en Actas XXI Jornadas de Historia Económica, Caseros, 2008.

CANADÉ, Juan. Las correcciones de los mostos, 1912.

CANTARELLI, Andrea. Avance del frente pionero en San Rafael y la conexión ferroviaria con Mendoza en 1903. IN: XX Jornadas de Historia Económica, Mar del Plata, UNMdP, 2006.

. "La inmigración italiana y el inicio de la vitivinicultura moderna en San Rafael, Mendoza. Siglos XIX- XX", en Actas V Jornadas de Investigación y Debate "Trabajo, propiedad y tecnología en la Argentina Rural del siglo XX", U.N.Quilmes, Bernal, 2008.

CAMPI, Daniel y RICHARD-JORBA, Rodolfo. Transformaciones sociales y productivas, espaciales y sociales en la Argentina extrapampeana. Tucumán y Mendoza entre 1850 y 1890. Boletín Americanista, Barcelona, Universidad de Barcelona, 2004, $\mathrm{n}^{\circ} 54$.

COZZANI DE PALMADA, Rosa. Sociedad y espacios de migración: italianos en Argentina y Mendoza. Mendoza: Ediunc, 1997.

CUETO, Adolfo. Participación del italiano en el campo económico-laboral mendocino (1870-1930). Revista del Ceider, $\mathrm{n}^{\circ}$ 5, Mendoza, UNCu, 1990.

DÍAZ ARAUJO, Edgardo e Ivaro, María José. Vitivinicultura y derecho. Buenos Aires: Dunken, 2006.

FONTANA, Esteban. La educación media superior mendocina. Contribuciones para la historia de Mendoza, Mendoza, Fac. Filosofía y Letras, UNCu, 1969.

FRESIA, Ariel. Religión, educación y vida cotidiana (Siglo XX). Buenos Aires: Dunken, 2005.

GARGIULO, Julieta. y BORZI, Agustín. Il vino si fa cosi. Mendoza: Polo Rossi, 2004.

GRACIANO, Osvaldo. Universidad y economía agroexportadora. El perfil profesional de los ingenieros agrónomos, 1910- 1930. IN: GIRBAL DE BLACHA, Noemi (dir). Agro, universidad y enseñanza. Dos momentos de la Argentina rural (1910-1955). La Plata: UNLP, 1998. 
El agro pampeano en el pensamiento universitario argentino. Las propuestas de los ingenieros agrónomos de la Universidad Nacional de La Plata, 1906- 1930. Cuadernos del PIEA, Fac. de Ciencias Económicas, UBA, n ${ }^{15}$, 2001.

- Los caminos de la ciencia. El desarrollo inicial de las Ciencias Agronómica y Veterinarias en Argentina, 1860- 1910. Signos Históricos, Univ. Autónoma Metropolitana-Iztapalapa, 2004, $\mathrm{n}^{0} 12$.

GUIBOURDENCHE DE CABEZAS, Marta. San Rafael visto a través de la estadística. Separata de la Fac. Cs. Económicas, UNCu, mayo-agosto de 1956.

GUTIÉRREZ, Talía. Proyectos educativos de orientación productiva. La enseñanza agrícola en la provincia de Buenos Aires y la Región pampeana, 18901930. Estudios de Historia Rural IV. Estudios /Investigaciones, No 27, 1996.

. Enseñanza agrícola y medio ambiente en la Región Pampeana, 19101955. Revista THEOMAI, Buenos Aires, $\mathrm{n}^{0}$ 2, segundo semestre 2000.

. Educación, agro y sociedad (1897-1955). Bernal: UNQ, 2007.

LACOSTE, Pablo. El vino del inmigrante. Mendoza: Universidad de Congreso, 2003.

LENIS, María y MOYANO, Ricardo. Modernizar el agro. La industria azucarera tucumana ante la crisis del mosaico. IN: Actas de las Primeras Jornadas de Jóvenes Investigadores, UNT-AUGM, 2006 (en CD).

MAROTTA, Pedro. La educación de los agricultores por los agrónomos regionales. Boletín $n^{o}$ 62, Buenos Aires, Talleres Gráficos del Ministerio de Agricultura, 1916.

MARTÍN, José Francisco. Estado y empresas. Mendoza: Ediunc, 1992.

MATEU, Ana y STEIN, Steve, "Diálogos entre sordos. Los pragmáticos y los técnicos en la época inicial de la industria vitivinícola argentina", en Historia Agraria, Universidad de Murcia, $\mathrm{n}^{\circ}$ 39, 2006.

(dir.), El vino y sus revoluciones. Una antología histórica sobre el desarrollo de la industria vitivinícola argentina, Mendoza, EDIUNC, 2008.

MENDONÇA, Sônia de. Agronomia e poder no Brasil. Rio de Janeiro: Vicio de leitura, 1998.

. Agronomía, agrónomos y estado en Brasil: organizaciones y disputas (1930- 1961). Mundo Agrario. Revista de estudios rurales, UNLP, $\mathrm{n}^{0}$ 9, segundo semestre 2004.

. Estado y enseñanza agrícola en Brasil: de la dimensión escolar al extensionismo-asistencialismo (1903-1950). IN: GIRBAL-BLACHA, Noemi y MENDONÇA, Sônia de (dir.). Cuestiones agrarias en Argentina y Brasil. Conflictos sociales, educación y medio ambiente. Buenos Aires: Prometeo, 2007.

RICHARD-JORBA, Rodolfo. Estado y empresarios regionales en los cambios económicos y espaciales. La modernización en Mendoza (1870- 1910). Siglo XIX Cuadernos de Historia, Univ. Autónoma de Nuevo León, no 10, 1994.

. Poder, economía y espacio en Mendoza (1850-19oo): del comercio ganadero a la agroindustria vitivinícola. Mendoza: Fac. de Filosofía y Letras, 
$\mathrm{UNCu}, 1998$.

"El mercado de trabajo vitivinícola en Mendoza y los nuevos actores. El 'contratista de viña': aproximación a un complejo sistema de empresarios y trabajadores, 1880-1910", en Revista Interdisciplinaria de Estudios Agrarios, IIHE-FCEconómicas, UBA, No 18, 2003.

. ¿Echar raíces o hacer la América? Un panorama de la inmigración europea hacia la región vitivinícola argentina y algunos itinerarios económicos en la provincia de Mendoza, 1850- 1914. Amérqie Latine Histoire e Mémoire. Les Cahiers ALHIM, $\mathrm{n}^{\circ}$ 9, 2004. Disponible en: <http://alhim.revues.org/index435.html> (Fecha de consulta: 11/o3/2006).

y PÉREZ ROMAGNOLI, Eduardo. La década de 1870 en Mendoza: etapa de reorientación de la economía y del espacio hacia el dominio vitivinícola. Boletín de Estudios Geográficos, Fac. Filosofía y Letras, UNCu, n ${ }^{0}$ 88, 1992.

. El proceso de modernización de la bodega mendocina (1860- 1915). Ciclos, UBA, año IV, vol. IV, $\mathrm{n}^{0} 7,2^{\circ}$ semestre 1994.

Richard-Jorba,. Rodolfo, et all. La región vitivinícola argentina (1870-1914). Bernal: UNQ, 2006.

RODRÍGUEZ VÁZQUEZ, Florencia. La difusión de conocimientos vitivinícolas en Mendoza: La educación formal y el aporte de la prensa (1890-1910). IN: IV Jornadas Interdisciplinarias de Estudios Agrarios y Agroindustriales, Bs. As., Fac. Cs. Económicas, UBA, 2005.

La transferencia tecnológica: el rol de los agentes formales y no formales en la difusión de conocimientos aplicados a la industria vitivinícola en la provincia de Mendoza (1910- 1914). IN: XX Jornadas de Historia Económica, Mar del Plata, UNMdP, 2006.

- La modernización agrícola en Argentina y los establecimientos educativos de orientación productiva: el aporte de la Escuela Nacional de Vitivinicultura (1900-1920). Revista Historia Regional, Instituto Superior del Profesorado $n^{0} 3$ "Eduardo Lafferriere", Rosario, n 26, 2008.

- Estado y modernización vitivinícola en Mendoza (Argentina): el aporte de los técnicos extranjeros. 1880- 1900, Territorios del Vino, $\mathrm{n}^{\circ}{ }_{2}$, Universidad de la República, Montevideo, 2008. Disponible en: <http://www.fhuce.edu.uy/academica/ceil-ceiu/ceil/Revista2TV >

SANJURJO DE DRIOLLET, Inés. La organización político- administrativa de la campaña mendocina en el tránsito del antiguo régimen al orden liberal. Buenos Aires: Instituto de Investigaciones de Historia del Derecho, 2004.

- Frontera indígena y colonias agrícolas en las localidades del sur de Mendoza entre 1854 y 1916. IN: RICHARD-JORBA, Rodolfo et all. La región vitivinícola argentina: Bernal: UNQ, 2006.

SUÁREZ, Leopoldo. Contribución a los estudios ampelográficos en la provincia de Mendoza. Mendoza, 1911.

Colaboração recebida em 25/03/2009 e aprovada em 25/05/2009. 